\title{
Unstable Path Routing in Urban-Scale WSN
}

\author{
Farrukh Mirza \\ Distributed Systems Group \\ School of Computer Science \\ and Statistics \\ Trinity College Dublin \\ Ireland \\ mirzaf@cs.tcd.ie
}

\author{
Mélanie Bouroche \\ Distributed Systems Group \\ School of Computer Science \\ and Statistics \\ Trinity College Dublin \\ Ireland
}

Melanie.Bouroche@cs.tcd.ie Vinny.Cahill@cs.tcd.ie

\author{
Vinny Cahill \\ Distributed Systems Group \\ School of Computer Science \\ and Statistics \\ Trinity College Dublin \\ Ireland
}

\begin{abstract}
Wireless sensor networks (WSNs) may suffer from congestion at the nodes near the sink, and partition due to the failure of crucial nodes. In urban environments, mobile devices, such as vehicles and smart phones, present in the vicinity of the sensor field could be opportunistically used for data forwarding. Such devices, controlled by third parties, introduce paths that may appear for only very small intervals.

This paper discusses how exploiting such unstable paths to shift the routing-related processing and communication load to more capable mobile devices can alleviate traffic congestion, improve fault tolerance and reduce WSN energy consumption.
\end{abstract}

\section{Categories and Subject Descriptors}

C.2.2 [Computer Communications Networks]: Network Protocols-Routing Protocols; C.2.1 [Computer Communications Networks]: Network Architecture and DesignWireless Communication

\section{General Terms}

Theory, Design, Unstable paths

\section{Keywords}

Unstable Path Routing, Urban Scale WSN, Data forwarding via participatory devices

\section{INTRODUCTION}

In large scale WSN, a number of challenges are faced, such as energy conservation, traffic congestion, fault tolerance, data delivery ratio, timeliness and scalability, among others. In an urban environment, individually-owned mobile devices that may have overall better resources may be present in the vicinity of a WSN. These mobile devices have the potential to forward data on behalf of the WSN. This research contributes to the routing domain of sensor networking by

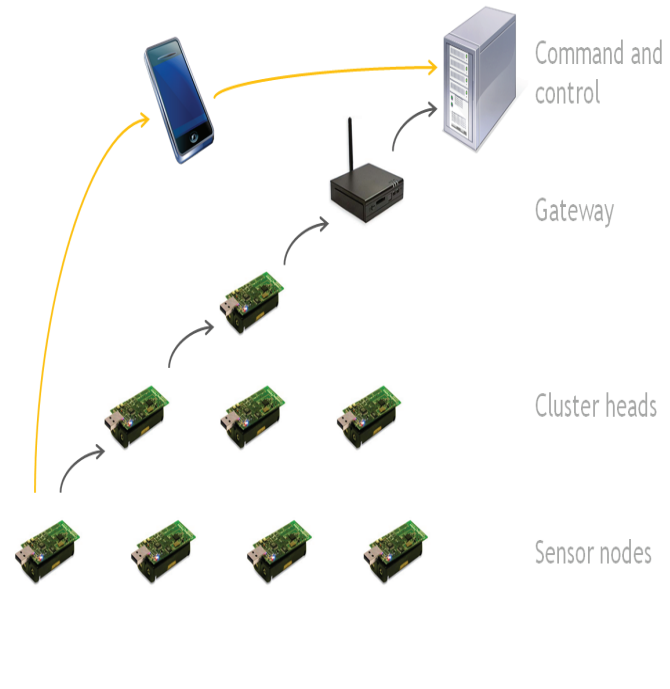

Stable, energy-costly route

_ Unstable, energy-efficient route

Figure 1: Exploiting unstable paths via mobile device.

exploring the possibility of taking advantage of such mobile devices, for data forwarding towards the destination in an opportunistic and dynamic manner (Figure 1). In doing so, the data forwarding (routing) load can be shifted away from resource constrained sensor nodes towards more capable mobile devices. Network congestion in particular areas may also be mitigated due to the availability of more capable mobile devices for data forwarding. This strategy may improve fault tolerance by bridging potential network partitions. In this way, energy consumption and processing load due to packet forwarding requests, at intermediate sensor nodes, can also be reduced. Third-party mobile devices could also potentially reduce end-to-end message latency because of the availability of higher transmission rates and better radios, resulting in over-all better timeliness. Finally such mobile devices may have more computational, storage and communication resources, achieving overall better throughput.

The paths introduced by these mobile devices, however, are 
unstable, i.e., they appear and disappear with the movement of mobile devices, or when a user turns on or off the radio responsible for communicating with the WSN. The instability of these paths may increase the risk of data loss. A trade-off may exist between energy conservation, end-toend delay reduction, throughput, congestion avoidance and risk of data loss. In addition, completely reliable route performance history may not be available, and acquisition of route information with absolute certainty will increase the control overhead. Therefore, devices will need to make decisions based on very limited information available locally, while still meeting application requirements.

A device differentiation mechanism is also required to select the next hop according to its ability to serve the network. In an urban environment, different types of available devices can be classified according to their generic capabilities such as battery, radio, mobility etc. Stationary sensor nodes have limited and non-rechargeable batteries and can communicate only over short-range radio, e.g., IEEE 802.15.4 [8]. Mobile devices (laptops and smartphones) have limited but rechargeable batteries, can communicate over short-range as well as long-range radio, e.g., IEEE 802.16 [9] and show both walking and vehicular speeds (user in a car). Vehicles and stationary line-powered devices can have unlimited energy reserves. Vehicles may also exhibit varying speeds. By connecting a compatible radio, mobiles and vehicles can communicate directly with WSN. In addition, the devices can also be differentiated according to the roles that they perform. A sink requests data from the WSN, a gateway connects a WSN with some other network such as Internet, and a relay forwards data within the same network. A device may also change its role or may acquire additional role(s), e.g., if a user requests data from the WSN, a mobile relay may suddenly also become a sink. Therefore, this mechanism should support a vast variety of devices that dynamically change their roles and characteristics.

This research is closely related to and complements EMMON [14], having a similar network architecture and relying on COTS $^{1}$ components. The EMMON project shows promising results in achieving scalable and time-bounded communication. This research is expected to further enhance those results, by bypassing a number of hops when the network depth increases, which otherwise results in a linear increase of end-to-end latency (Figure 1), and by potentially reducing congestion at nodes that have many children. In this way, we expect to further improve scalability, energy conservation and end-to-end latency.

The essential problem to be solved to successfully exploit unstable paths is, given the mobility patterns of sink, relay and gateway, how can the best next hop be selected in a WSN in order to move data forwarding load to more capable devices and in doing so obtain the right trade off in terms of energy consumption, latency and risk of data loss.

The remainder of the paper is organised as follows. Section 2 gives an overview of the related work, Section 3 identifies and discusses challenges that need to be addressed to design a routing protocol that can efficiently take advantage

\footnotetext{
${ }^{1}$ Commercially available Off-The-Shelf
}

of unstable paths and finally Section 4 concludes this paper.

\section{RELATED WORK}

The majority of the protocols that consider the interaction of stationary WSN with mobile devices, only support delivering data from the WSN to mobile sinks. These protocols hide movement of the mobile sink from the WSN by employing a sensor node as proxy for the mobile sink, which is typically the nearest node to the mobile sink. These protocols first establish routes towards the initial proxy node, so that the data flows towards the proxy node. The proxy node then forwards the data to the mobile sink. EARM, SEAD, IAR and TTDD $[1,10,11,18]$ extend existing routes from the proxy node towards the mobile sink via other sensor nodes, as the mobile sink moves away. The existing routes are extended unless new routes are absolutely necessary and/or old routes become significantly inefficient. Kusy et al. [12] take a different approach by continuously changing the proxy node with the movement of the mobile sink, by predicting the movement pattern of mobile sinks and identifying the future proxy nodes before they are needed. All these protocols only aim to deliver data to the mobile sinks but do not shift routing load away from sensor nodes.

ROME [3] supports mobile devices as relays, by creating logical gradients converging towards a stationary sink. ROME, however, only forwards data via mobile devices when they are not in motion, hence using them only a fraction of the time.

The closest related routing protocols to this work are those that differentiate among devices and favour more capable devices. DEAR [2] differentiates among devices based on their energy reserves by classifying them into only two categories, i.e., battery powered and line powered. As the more capable devices are line-powered, they are not mobile. The MC-Routing protocol [6] also classifies devices into only two categories. However, the classification is based on transmission range, data rate and processing capabilities. It creates a logical cellular structure and assumes at least one backbone (more capable) node in each cell. The backbone nodes are also assumed to communicate directly with other backbone nodes in adjacent cells. Similarly, hierarchical routing protocol such as HOLSR [16] and HCB [17] can also differentiate among devices, however, based solely on their number of radios. In hierarchical protocols, the traffic is routed towards more capable devices when the destination is outside the cluster.

These protocols rely on route discovery procedures, mostly initiated by the source node that may be resource constrained. In highly dynamic environments, due to the appearance and disappearance of devices, the route discovery procedures will be initiated frequently, increasing the control overhead. In addition the prospective route may break before any data has reached its destination, rendering the approach unusable.

\section{DESIGN CHALLENGES}

Solving the problem described in Section 1 essentially requires answering the question of how the forwarding capability of a newly-arrived device can be judged and compared to that of existing neighbours, in a highly dynamic environ- 


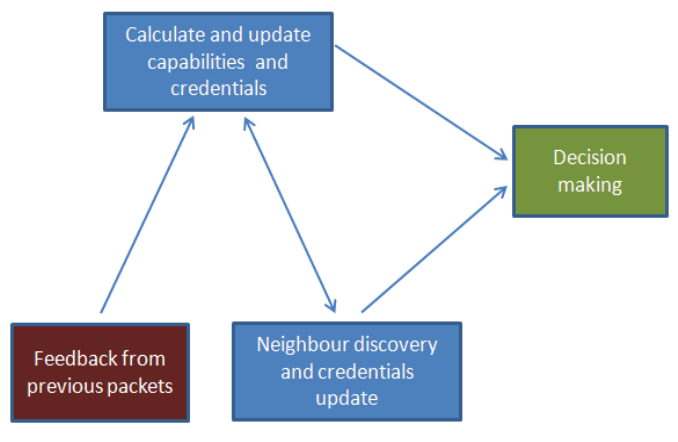

Figure 2: Solution components.

ment. Keeping in mind the unstable nature of the prospective routes, the protocol cannot rely on explicit route discovery procedures (Section 2). Similarly, pure geographic routing approach may not be viable, because more capable devices may not be the closest ones to the destination. Hence, the routing protocol should be able to react fast to the changing environment, as well as being able to identify and favour more capable devices.

The scale of the problem considered here, as well as the number of parameters in consideration mean that it is intractable to create a model of the environment. Indeed, the effect of selecting a particular next hop cannot be fully predicted as all operating conditions cannot be predefined and hence the correct behaviour cannot be decided at the design time. Reinforcement learning (RL) [13] is considered particularly suitable for such problems [15], and we are therefore exploring how it could be used in this context. However, it is not completely apparent whether RL approach can react fast enough to cater to the requirements of such a highly dynamic environment. In addition, the set of actions (the set of individual devices acting as data forwarders) may always be changing in contradiction to typical RL problems. Hence, the solution is inspired by RL, however, some customization is required in accordance with the environment. RL has been previously used in the packet routing domain $[5,7,4]$, however some notion of reaching a stable state is assumed, contrary to this research where the stable state remains elusive.

The problem is decomposed into four major components (Figure 2) and formulated as a RL problem. The arrows in Figure 2 show dependencies among different components. Each of the components are discussed below along with some of the design challenges that they incur.

\subsection{Decision Making}

This component is concerned with the next hop selection, enabling competition among potential forwarders, corresponding to action selection in RL. Depending on the action selection policy, this component may occasionally allow the current-hop (node holding the packet) to explore sub-optimal actions [13], allowing better routing opportunities to be explored in a dynamic environment, however, at the expense of overhead. The placement of this component is crucial as it dictates the design of the solution. This component can either be placed at newly-arrived devices that wish to take over the packet and become the next hop, or at the current hop that wishes to find a potential next hop.

In the former case, the packet will be voluntarily taken over and forwarded towards the destination. The destination may receive multiple copies of the same packet. This mechanism places most of the routing-related load at the more capable devices. It increases the data delivery probability as multiple copies of the same packet will travel towards the destination. This, however, also increases the network traffic.

In the latter case, the newly-arrived device advertises itself along with its ability to deliver data to the destination. Taking advantage of the newly-arrived device is at the discretion of the current hop. This mechanism ensures that only a single copy of the packet reaches the destination, at the expense of slightly increasing processing load at the less capable devices. It reduces the overall traffic in the network, but also the data delivery probability.

\subsection{Calculate and Update Capabilities and Cre- dentials}

As discussed in Section 1, device differentiation and assessment mechanisms are needed. A device's packet delivery ability assessment can be divided into device's own capabilities dictated by the resources and roles that it possesses, and the device's credentials that reflect the "goodness" of a device for delivering packets to their destinations.

Device capability reflects the tasks that the device can perform. A device that has an active long-range radio can send packets to Internet destinations. Similarly, a device with rechargeable or un-limited energy reserves may forward packets more frequently.

Device credentials specify the trustworthiness and/or feasibility to deliver packets to a certain destination or type of destination. They will improve as a device successfully delivers packets to their destinations. They not only depend on performance statistics, but also on the current environment. The credentials can be further divided into their long-term and short-term components.

In terms of RL, this component will consist of a state-value function (e.g., a Q-value function), influenced by a number of factors (discussed below). Different weights can be associated with information from the current environment and past performance. Weighted parameters can allow the application to change its delivery priority, however, they reduce the usability of the protocol.

\subsubsection{Rewards from feedback}

Rewards are received via feedback from downstream nodes, as a device participates in data forwarding. Performance of next hops can be evaluated from these rewards. They influence both short-term and long-term credentials.

\subsubsection{Current state}

The current state is influenced by the short-term component of the device credentials. Factors such as remaining energy, 
distance to destination and velocity contribute to the current state. Device capabilities such as active radios also influence the current state.

In a highly dynamic environment, acquiring the route health information is very costly. Therefore, a (mobile) relay may store, update and advertise its own credentials. This strategy will contribute in shifting the routing-related load towards more capable devices. Calculating credentials at the potential next hop may, however, raise trust issues such that the next hop may provide false or unreliable information.

\subsubsection{Past statistics}

Past statistics will be recorded and used as the device participates in data forwarding, influencing the long-term component of credentials. Successful deliveries to particular or similar destinations and frequency of visiting locations or areas near destinations, become part of these statistics. Information reliability however, deteriorates with time.

\subsubsection{Neighbourhood}

Neighbourhood's capabilities and credentials influence the short-term component of device credentials. The update mechanism is discussed in section 3.3 .

\subsection{Neighbour discovery and credential update}

This component is concerned with the neighbourhood discovery and the credential propagation. More capable devices will scan radio channel and discover neighbourhood, to shift load from resource constrained devices. The newly-arrived devices will, however, have to synchronize with the waking period of the sensor nodes.

Newly-arrived mobile devices will promiscuously listen to and analyze all the packets in their vicinity. By doing so, a mobile device can assess itself to deliver the same packet and will become aware of its neighbourhood.

A device can either influence its neighbourhood by periodically advertising its abilities, or be influenced by its neighbourhood by promiscuously listening to the packet transmissions in its vicinity. In the former case, a device will discover all neighbours, increasing the memory requirements of the protocol. In the latter case, a device will discover only those neighbours that are currently forwarding data and none if no data is currently transmitted.

\subsection{Feedback}

A device will acknowledge the reception of a data packet to the previous hop. The performance estimate is obtained from this acknowledgement containing the expected reward. This estimate will improve with experience and should converge towards an accurate reward.

The first accurate reward is received by the hop n- 1 from the final destination (n) upon reception of the packet. The next time a packet is delivered to the same destination; hop n-2 receives an accurate reward from hop $\mathrm{n}-1$, and so on. This mechanism allows intermediate nodes to become aware of their performance and make better decisions along the way. If congestion is detected, the previous hop can be advised to find alternative routes. This process will, however, take several iterations before all the nodes are fully aware about their real performance, reducing the protocol's reactivity. Optimizing this mechanism to react fast is crucial in this research.

\section{CONCLUSIONS}

This paper discussed our research in-progress that explores taking advantage of unstable routes created by third-party mobile devices and assessment of device's data forwarding ability in a highly dynamic environment. It also discussed the solution design approach for a routing protocol that can efficiently move the routing load away from less capable devices, in addition to the challenges faced while designing the protocol. Using these unstable paths promise great rewards in terms of energy conservation, end-to-end latency reduction, throughput improvement and fault tolerance improvement. The unstable nature of these routes, however, may also increase the risk of data loss.

Currently, the mathematical basis upon which credentials can be updated and the state-value function can be influenced is being explored, so that the solution reacts quickly to the changing environment. Also the design of evaluation criteria is in progress along with the detailed design and specification of different mechanisms for components involved in the envisaged routing protocol.

The next steps in this research involve the evaluation of the routes created by third-party mobile devices through an implementation of the routing protocol on a WSN testbed.

\section{ACKNOWLEDGMENTS}

This work is supported by Enterprise Ireland, the National Development Plan (Ireland) and the European Union via the ARTEMIS Joint Undertaking, under grant agreement n. 100036 .

\section{REFERENCES}

[1] K. Akkaya and M. Younis. Energy-aware routing to a mobile gateway in wireless sensor networks. In Global Telecommunications Conference Workshops (GlobeCom Workshops) 2004, pages 16-21. IEEE, 2004.

[2] A. Avudainayagam, W. Lou, and Y. Fang. DEAR: A Device and Energy Aware Routing protocol for heterogeneous ad hoc networks. Journal of Parallel and Distributed Computing, 63(2):228-236, 2003.

[3] S. Basagni, M. Nati, C. Petrioli, and R. Petroccia. Rome: Routing over mobile elements in wsns. In Global Telecommunications Conference, 2009. GLOBECOM 2009. IEEE, pages 1-7. IEEE, 2010.

[4] J. Boyan and M. Littman. Packet routing in dynamically changing networks: A reinforcement learning approach. Advances in neural information processing systems, pages 671-678, 1994.

[5] J. Dowling, E. Curran, R. Cunningham, and V. Cahill. Using feedback in collaborative reinforcement learning to adaptively optimize MANET routing. Systems, Man and Cybernetics, Part A: Systems and Humans, IEEE Transactions on, 35(3):360-372, 2005.

[6] X. Du, D. Wu, W. Liu, and Y. Fang. Multiclass routing and medium access control for heterogeneous 
mobile ad hoc networks. Vehicular Technology, IEEE Transactions on, 55(1):270-277, 2006.

[7] A. Forstert and A. Murphy. FROMS: Feedback routing for optimizing multiple sinks in WSN with reinforcement learning. In Intelligent Sensors, Sensor Networks and Information, 200\%. ISSNIP 2007. 3rd International Conference on, pages 371-376. IEEE, 2008.

[8] IEEE802.15.4. Part 15.4: Wireless Medium Access Control (MAC) and Physical Layer (PHY) specifications for Low-Rate Wireless Personal Area Networks (WPANs), September 2006.

[9] IEEE802.16. Part 16: Air Interface for Fixed and Mobile Broadband Wireless Access Systems, Amendment 2: Physical and Medium Access Control Layers for Combined Fixed and Mobile Operation in Licensed Bands and Corrigendum 1, 2005.

[10] H. Kim, T. Abdelzaher, and W. Kwon. Minimum-energy asynchronous dissemination to mobile sinks in wireless sensor networks. In Proceedings of the 1st international conference on Embedded networked sensor systems, pages 193-204. ACM, 2003.

[11] J. Kim, J. In, K. Hur, J. Kim, and D. Eom. An intelligent agent-based routing structure for mobile sinks in WSNs. Consumer Electronics, IEEE Transactions on, 56(4):2310-2316, 2010.

[12] B. Kusy, H. Lee, M. Wicke, N. Milosavljevic, and L. Guibas. Predictive qos routing to mobile sinks in wireless sensor networks. In Information Processing in Sensor Networks, 2009. IPSN 2009. International Conference on, pages 109-120. IEEE, 2009.

[13] R. Sutton and A. Barto. Reinforcement learning: An introduction. The MIT press, 1998.

[14] S. Tennina, M. Bouroche, P. Braga, R. Gomes, M. Alves, F. Mirza, V. Ciriello, G. Carrozza, P. Oliveira, and V. Cahill. Emmon: A wsn system architecture for large scale and dense real-time embedded monitoring. In Embedded and Ubiquitous Computing (EUC), 2011 IFIP 9th International Conference on, pages 150-157. IEEE, 2011.

[15] G. Tesauro. Reinforcement learning in autonomic computing: A manifesto and case studies. IEEE Internet Computing, pages 22-30, 2007.

[16] L. Villasenor-Gonzalez, Y. Ge, and L. Lament. HOLSR: a hierarchical proactive routing mechanism for mobile ad hoc networks. Communications Magazine, IEEE, 43(7):118-125, 2005.

[17] Y. Xia, C. Yeo, and B. Lee. Hierarchical Cluster Based Routing for Highly Mobile Heterogeneous MANET. School of Computer Engineering, Nanyang Technological University, Singapore, 2009.

[18] F. Ye, H. Luo, J. Cheng, S. Lu, and L. Zhang. A two-tier data dissemination model for large-scale wireless sensor networks. In Proceedings of the 8th annual international conference on Mobile computing and networking, page 159. ACM, 2002. 